\title{
URBANISMO EXPOSITIVO EXPERIMENTADO DESDE LA MODERNIDAD MIESIANA
}

Laura Lizondo

Profesora, Escola Tècnica Superior d'Arquitectura, Universitat Politècnica de València

José Santatecla-Fayos

Profesor, Escola Tècnica Superior d'Arquitectura, Universitat Politècnica de València

Ignacio Bosch-Reig

Catedrático, Escola Tècnica Superior d'Arquitectura, Universitat Politècnica de València

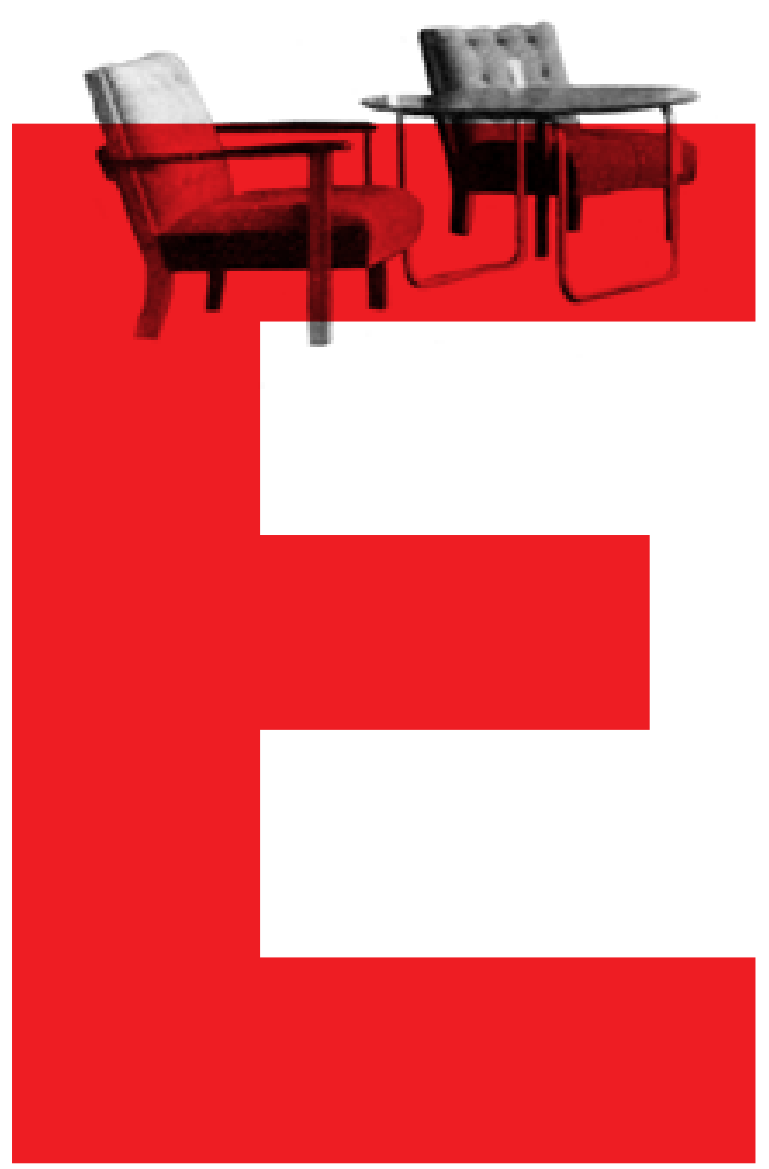

LA COLONIA WEISSENHOF Y LA MUESTRA DIE WOHNUNG UNSERER ZEIT a modernidad es lo transitorio, lo fugitivo, lo contingente (Baudelaire, 1863). Las palabras de Baudelaire ayudan a introducir directamente este discurso. Y es que las mayores manifestaciones de modernidad se han producido en los escenarios efímeros y volátiles. Todo aquello que no responde a lo eterno e inmutable se convierte en pretexto para la experimentación. Nunca ha sido diferente, menos en arquitectura. Cuando el arquitecto diseña un proyecto temporal, no sometido a las leyes estrictas de la permanencia y en donde no existe el peligro de dañar irreversiblemente el entorno en que se construye, el subconsciente se focaliza en dar una respuesta significada, incluso en ocasiones visionaria. Así, "las exposiciones han sido el lugar para los proyectos más experimentales" (Colomina, 2009), el lugar que ha servido de antesala de muchas arquitecturas permanentes. Sin pensar demasiado, nos vienen a la cabeza innumerables ejemplos; desde los innovadores edificios construidos para las Exposiciones Universales hasta los Pabellones Experimentales, como el Pabellón de Cristal de Bruno Taut, presentado en la Exposición de Colonia de 1914, el Pabellón de L'Esprit Nouveau de Le Corbusier y Pierre Jeanneret para la Exposition Internationale des Arts Décoratifs et Industriels Modernes de París de 1925 o el Pabellón Alemán proyectado por Mies van der Rohe para la Exposición Internacional de Barcelona de 1929. Todos ellos formaron parte de una estrategia de conjunto; edificios que aunque desaparecieron parcialmente en el instante en el que se desmontaron, condicionaron las directrices edilicias y urbanísticas futuras de la ciudad que les acogió.

Bajo este mecanismo experimental, las exposiciones se convirtieron en el escenario arquitectónico y urbanístico donde gran parte de los arquitectos de la vanguardia dieron respuesta a sus preocupaciones proyectuales, muchas veces "rebasando los límites de la arquitectura" (Pérez Oyarzun, 2006). Pero en el caso de Mies van der Rohe, esta experimentación no fue puntual, sino continuada durante algo más de una década. Su búsqueda abarcó el ámbito del edificio y del planeamiento, lo que implica, como consecuencia necesaria, la interesante consideración de los espacios intersticiales existentes entre estas dos escalas. Así lo atestiguan sus propuestas para la colonia de viviendas de la Weissenhof y la ordenación para la muestra Die Wohnung unserer Zeit. Dos arquitecturas expositivas que surgieron bajo el mismo pretexto y las mismas ideas iniciales, pero que difirieron enormemente en sus resultados; la permanencia contra la temporalidad y la diferenciación entre un emplazamiento real y un escenario cerrado y figurado, incluido dentro de una gran nave, marcarán las principales divergencias entre dos actuaciones que fueron el foco de atención de la crítica arquitectónica de la 
Palabras clave: Urbanismo, exposiciones, arquitectura efímera, Mies van der Rohe, Weissenhofsiedlung, Die Wohnung unserer Zeit.

Two urban projects by Mies van der Rohe, both exhibits, present a vision of modern architecture on the relationship between housing and the city. One is a permanent settlement and the other an ephemeral installation, built within an existing shed.

Keywords: Urbanism, exhibitions, ephemeral architecture, Mies van der Rohe, Weissenhofsiedlung, Die Wohnung unserer Zeit.

época. Ambas se construyeron como actuaciones urbanas unitarias a partir de la individualidad creativa de cada edificio y de cada arquitecto; ambas ambicionaron la continuidad espacial entre espacios públicos y privados, entre el concepto de mancomunidad y el de vivienda; ambas pretendieron dar respuesta a un nuevo estilo de vida que, en su mayor parte, no fue entendido ni por el público ni por la crítica, ...." porque lo convencional se disfruta sin criticarlo y se critica con aversión lo verdaderamente nuevo" (Benjamin, 1936).

\section{EDIFICIOS EXPOSITIVOS EN PAISAJES NATURALES.}

LA PERMANENCIA DE LA WEISSENHOFSIEDLUNG

La exposición Die Wohnung (La Vivienda, Stuttgart, 1927) se planteó como lugar de reflexión, donde dar respuesta al "cómo vivir" en la modernidad. De hecho, esta pregunta fue el lema de los carteles que anunciaban de la muestra -diseñados por Willi Baumeister y Karl Strab- y que reivindicaban la necesidad de un cambio, no sólo concerniente a los sistemas constructivos propios de la era de la máquina, sino también relativos al papel social e individual de la vivienda. Así lo evidencia la gran escala y el enérgico color del aspa que tacha la decoración clásica de la vivienda que da fondo a los carteles (fig. 01). Para mostrar convenientemente toda la experimentación realizada en torno a la vivienda, el DeutscherWerkbund, organismo promotor de la exposición, además de equipar una serie de salas expositivas -donde se mostraban materiales industriales, mobiliario, instalaciones técnicas o maquetas- propuso construir un asentamiento residencial permanente diseñado por arquitectos de toda Europa; una experiencia expositiva a escala 1: 1 donde poner en común toda la teoría de la arquitectura moderna internacional. La persona encargada de orquestar el planeamiento de conjunto fue Mies van der Rohe quien, como director artístico, diseñó la ordenación global de la parcela, escogió a los arquitectos que participarían en el diseño de las viviendas, marcó las directrices formales de los edificios y proyectó el único bloque plurifamiliar de la colonia, cuya escala y geometría definió el paisaje urbano resultante.

La idea germen en la materialización de la Weissenhof tuvo sus raíces en la tradición de las Ausstellunssiedlungen, más concretamente en el modelo establecido en la Künstler-Kolonie de Darmstadt, ideada en 1901 por el Duque Ernest Ludwig de Hesse y el arquitecto Josep María Olbrich (Pommer E Otto, 1991) (fig. 02). Ambas actuaciones se asemejan en la manera de situar la colonia en relación a la topografía. Al igual que Olbrich, Mies situó las viviendas en la zona más alta de la colina y dispuso el bloque de mayor densidad, diseñado por él, en el punto más elevado, actuando como hito del conjunto y límite referencial entre el paisaje construido y el paisaje natural. Sin embargo, el lenguaje arquitectónico de sendas propuestas no tenía nada en común; mientras que la Künstler-Kolonie recordaba los planteamientos clásicos, con trazados simétricos en relación con una plaza poligonal donde cada vivienda se levantaba aislada sin apenas espacio de esparcimiento, la primera propuesta de la Weissenhofsiedlung era propia de los planteamientos modernos, con una configuración orgánica, irregular y sin un centro específico; una propuesta cercana a los modelos anglosajones de "ciudad jardín" que abogaban por trazas curvilíneas adaptadas a la topografía. Así lo refleja la maqueta de arcilla de su primera propuesta (fig. 03).

Este primer plan, que únicamente definió los trazados urbanísticos y las masas cúbicas de los edificios, resultó en una gran complejidad y modernidad por el modo en que Mies agrupó las viviendas, interconectando unas con otras y aportando nuevas alternativas para los espacios comunes a ellas ${ }^{1}$. Rechazó generar una composición a base de filas estrictamente paralelas de alturas invariables, y adaptó la forma y altura de los edificios a la topografía y curvatura del planeamiento. Organizó los volúmenes y sus terrazas en una agrupación entrelazada, y no los consideró, según sus propias palabras, "paquetes separados". El documento 4.195 del Mies van der Rohe Archive muestra cómo edificios se solapaban unos con otros, confundiéndose los márgenes entre cada uno de ellos; espacios privados y al mismo tiempo compartidos que adelantaban un nuevo concepto de habitabilidad, parcelas superpuestas sin límites concretos. La única limitación formal establecida por Mies fue que las viviendas se construyesen con superficies exteriores planas y blancas, lo que incluía también a la quinta fachada. La ausencia de restricciones pretendía conseguir una unidad de conjunto que, simultáneamente, no sacrificara la libertad individual de los arquitectos participantes. Sin embargo, el proyecto de parcelas entrelazadas complicaba en gran medida el trabajo personal de cada proyectista, siendo inviable concebir las viviendas de manera independiente.

Además de las dificultades de diseño, el intento por romper la privacidad no fue bien aceptado ni por los profesores de la Hochschule de Stuttgart, Paul Bonatz y Paul Schmitthenner² ni por los miembros del Ayuntamiento, que interpretaron la ordenación como una amenaza a la propiedad privada. La propuesta tampoco fue respaldada por los modernos ortodoxos, en particular por

1 La idea de entrelazar fragmentos de masas cúbicas adaptadas a la topografía, y su consecuente reducción del espacio urbano tiene sus antecedentes en los planteamientos de la ciudad medieval y la ciudad islámica. La diferencia principal entre estas ordenaciones y la propuesta de Mies, radica en que mientras que en la antigüedad los espacios entrelazados fueron creados como ámbitos de uso privado, en la el urbanismo de Mies pretendían ser de uso público.

2 Tanto Bonatz como Schmitthenner publicaron sendos artículo en los diarios que hablaban sobre la exposición. Bonatz lo hizo en el diario Scwäbische Chronik de Stuttgart y denunció la propuesta subjetiva y dilentantista describiéndola como "un montón de cubos lisos dispuestos en múltiples terrazas horizontales que ascienden de un modo estrecho e incómodo por la pendiente". Schmitthenner lo hizo en el diario Süddeutsche Zeitung de Múnich, calificando el esquema como 'formalista y romántico'(Schulze, 1985:138). 


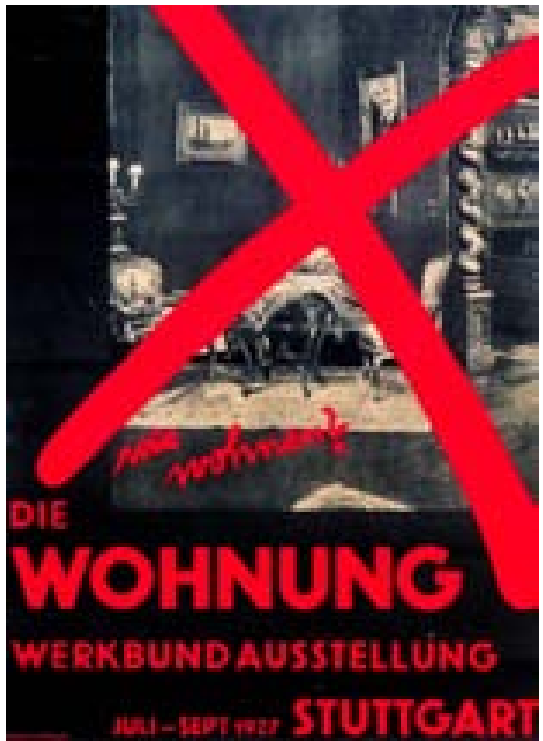

1. Anuncio de la Exposición Die Wohnung, Stuttgart, 1927. Diseño de Willi Baumeister y Karl Strab. Fuente: Vitra Design Museum. Mies van der Rohe. Arquitectura y Diseño en Stuttgart, Barcelona y Brno. Ministerio de Fomento; Fundación Carlos de Amberes; Skira, Madrid, 2001.
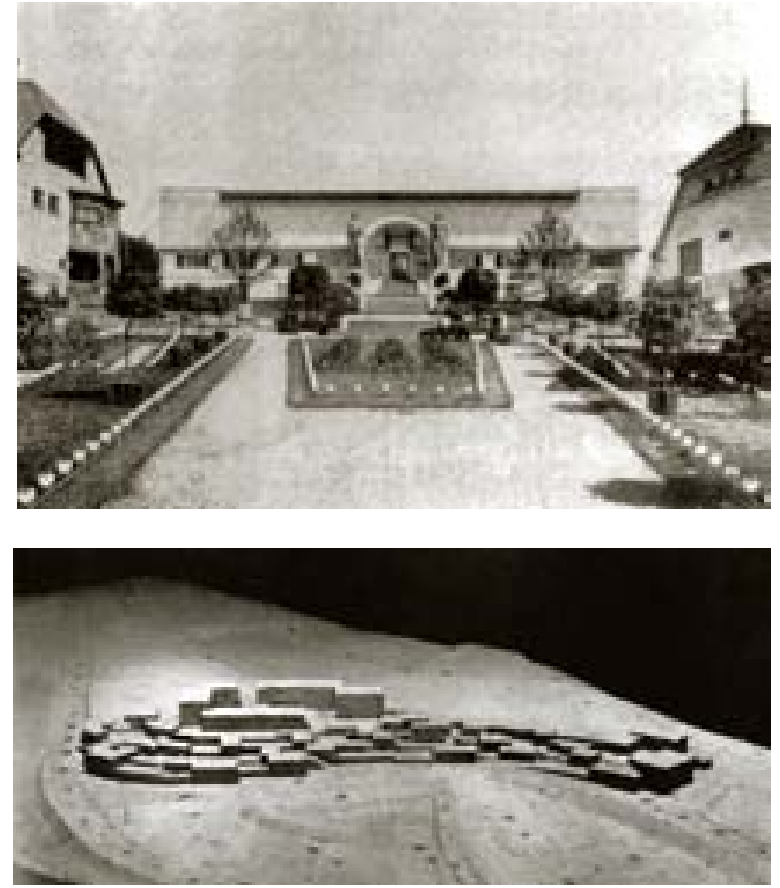

3. Maqueta de arcilla de la primera propuesta para la Weissenhof. Diseño de Mies van der Rohe.

Fuente: AA.VV. Mies in Berlin. Museum of Modern Art, Harry N. Abrams, Inc., Nueva York, 2001.

Propiedad de The Museum of Modern Art, The Mies van der Rohe Archive, Nueva York
2. Künstler-Kolonie, Darmstadt 1901. Diseño de Josep María Olbrich

Fuente: Pommer, R. y C. F. Otto. Weissenho 1927 and the Modern Movement in

Architecture. University Press, Chicago, 1991

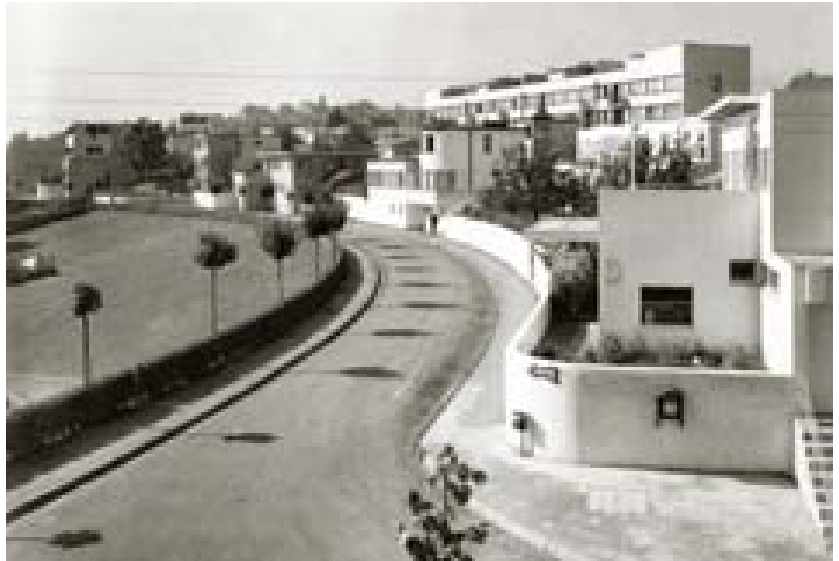

4. Fotografía de la propuesta construida para la Weissenhof. Diseño de Mies van der Rohe.

Fuente: Zimmerman, Claire. Mies van der Rohe 1886-1969. The structure of space. Taschen, Colonia, 2006.

Propiedad de The Museum of Modern Art, The Mies van der Rohe Archive, Nueva York.
Richard Döcker, compañero de Mies y participante de la exposición. Así lo expresó:

"Mezclar bloques de una, dos y tres alturas (...) es inorgánico, realizable, como mucho sólo parcialmente en planta, y por tanto nada objetivo. (...) Las viviendas están tan juntas y son tan variadas en cuanto a sus tamaños, formas y orientación, que el sol y las vistas dejan algunas de las habitaciones sin iluminación y ventilación y por tanto se requieren terrazas y jardines que se exceden del presupuesto". ${ }^{3}$

Tras las críticas y tras la decisión, por parte de las autoridades de Stuttgart, de convertir las unidades residenciales en permanentes, Mies tuvo que desarrollar un segundo plan que individualizara y definiera claramente los límites entre la propiedad privada y el espacio público de los solares, principalmente para facilitar su venta a particulares. El documento 4.91 del Mies van der Rohe Archive muestra un esquema que, aunque seguía siendo en forma de corona -elevándose de acuerdo a las curvas de nivel de la colina y rematado en la cima por el edificio de Mies- a diferencia del anterior no seguía el trazado curvilíneo de la calle principal, excepto en la esquina sureste (fig. 04). Tampoco los edificios se solapaban entre sí, sino que cada uno estaba claramente diferenciado dentro de unos límites parcelarios reales, tangibles e independientes. Aunque Mies había conseguido proyectar un conjunto unitario de escala urbana a partir de la individualidad lingüística de la pequeña escala, no pudo ver construidos sus ideales más sociales, basados en la abstracción entre los límites propiamente privados y los públicos, la frontera física e inmaterial donde el paisaje y el edificio confluyen (fig.05).

EDIFICIOS EXPOSITIVOS EN PAISAJES ARTIFICIALES. LA TEMPORALIDAD DE LA MUESTRA DIE WOHNUNG UNSERER ZEIT La exposición Deutsche Bauausstellung (Exposición de la Construcción Alemana, Berlín, 1931) se celebró en el recinto ferial de Reichskanzlerplatz de Berlín con el objetivo de exhibir la producción alemana en el sector de la arquitectura, el urbanismo y la industria de la construcción. Los patrocinadores de la muestra fueron la Asociación de la Industria de la Construcción Alemana y el Ayuntamiento de Berlín. El Deutscher Werkbund ${ }^{4}$ participó en calidad de gestor artístico, científico y social, pero teniendo un modesto papel y ningún poder de decisión en el desarrollo y las fases del evento. Aunque la primera intención de la organización fue construir una exposición de carácter permanente, la idea fue finalmente rechazada por motivos económicos. Una de las primeras secciones que sufrió el recorte presupuestario fue la muestra Die Wohnung unserer Zeit (La vivienda de nuestro tiempo), la cual pasó de ser un asentamiento permanente, como las Siedlungen, a una exposición temporal construida dentro de una de las salas expositivas que conformaban el recinto ferial. En un primer momento Otto Bartning fue la persona encargada de la dirección de la sección, pero en mayo de 1930, por motivos de salud, Bartning renunció en favor de Mies van der Rohe (Miller, 2007). El planteamiento de Mies desechó la propuesta inicial de Bartning, que abogaba por la convivencia de la vivienda progresista y la conservadora, reivindicando desde el primer momento una idea acorde con los nuevos tiempos, con la nueva arquitectura y con los requerimientos de las formas de vida modernas.

Las unidades residenciales del conjunto serían diseñadas por los miembros del Werkbund. Aunque el tamaño de las viviendas fuera a ser escala 1: 1 y el visitante pudiera experimentarlas como casas reales, su localización en el interior de una gran nave contenedor, su diseño experimental y la recreación artificial de los entornos, seguían recordando su condición de grandes maquetas, tal y como puede observarse en las fotografías que inmortalizaron la exposición (fig. 06). La idea predominante fue, de nuevo, la ansiada búsqueda miesiana por mostrar el nuevo concepto de vivienda y de comunidad, en donde la distinción entre lo público y privado no tenía un límite concreto y definido. Aunque "La Vivienda de

3 Richard Döcker en una carta a Mies van der Rohe datada el 18 de Mayo de 1926. Mies van der Rohe Archive. Werkbund Exhibition. Weissenhof. Folder 12.1. Item A

4 Asociación fundada por Herman Muthesius en 1907 que reunía arquitectos, artistas e industriales y que intentaba estimular el área de las Artes y Oficios alemana (Nota del ed.). 
5. Fotografía de la propuesta construida para la Weissenhof. Diseño de Mies van der Rohe. Fuente: AA.VV. Mies in Berlin. Museum of Modern Art, Harry N. Abrams, Inc. Nueva York, 2001. Propiedad de The Museum of Modern Art, The Mies van der Rohe Archive, Nueva York.
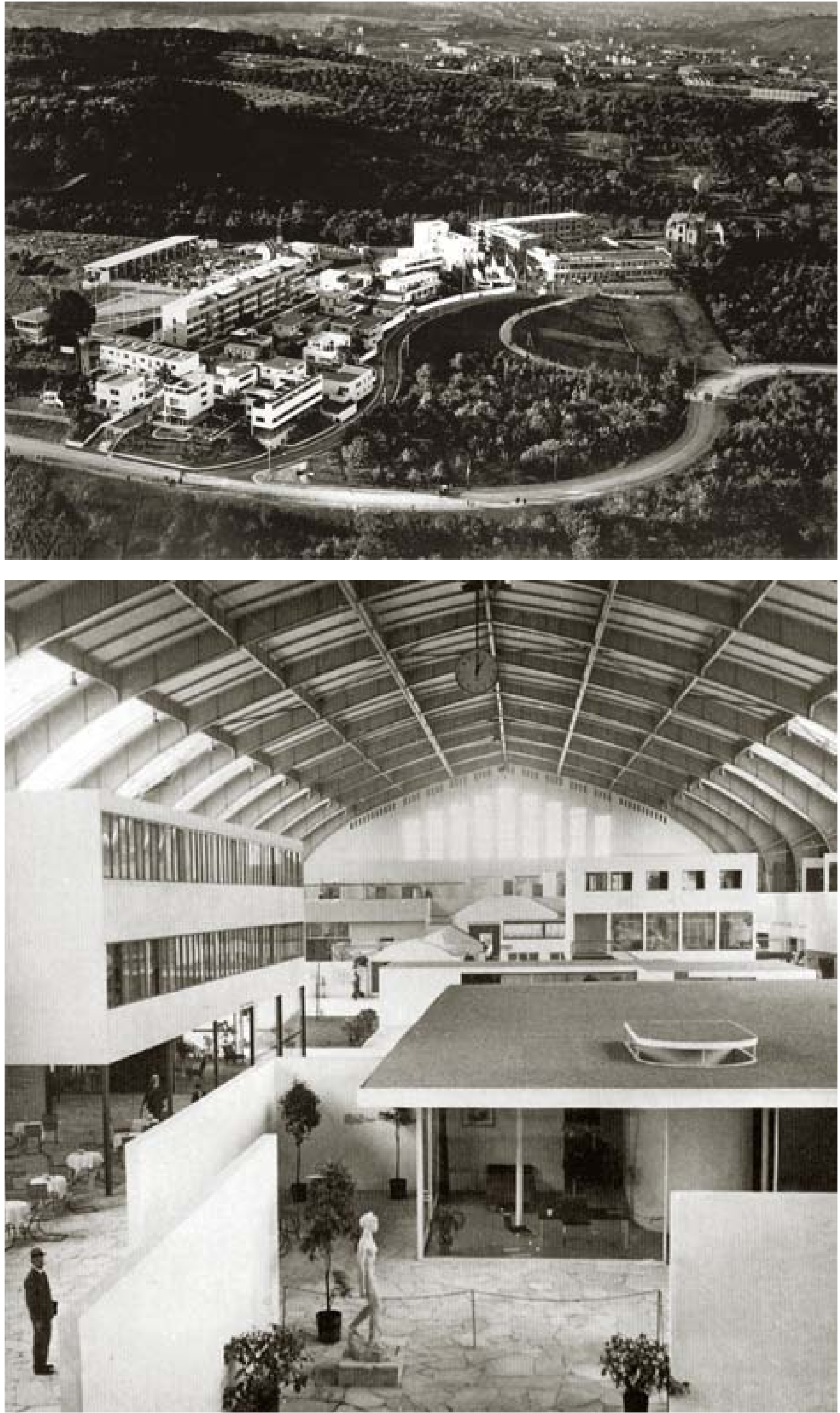

6. Fotografía del conjunto de la muestra Die Wohnung unserer Zeit. Exposición Deutsche Bauausstellung de 1931. Diseño de la ordenación de Mies van de Rohe. Vista desde la "Vivienda para un matrimonio sin hijos" Fuente: Von Ursel, H. y T. Pavel.

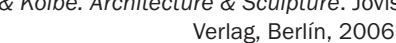
Propiedad de Stiftung Bauhaus Dessau VG Bild-Kunst Bonn. 


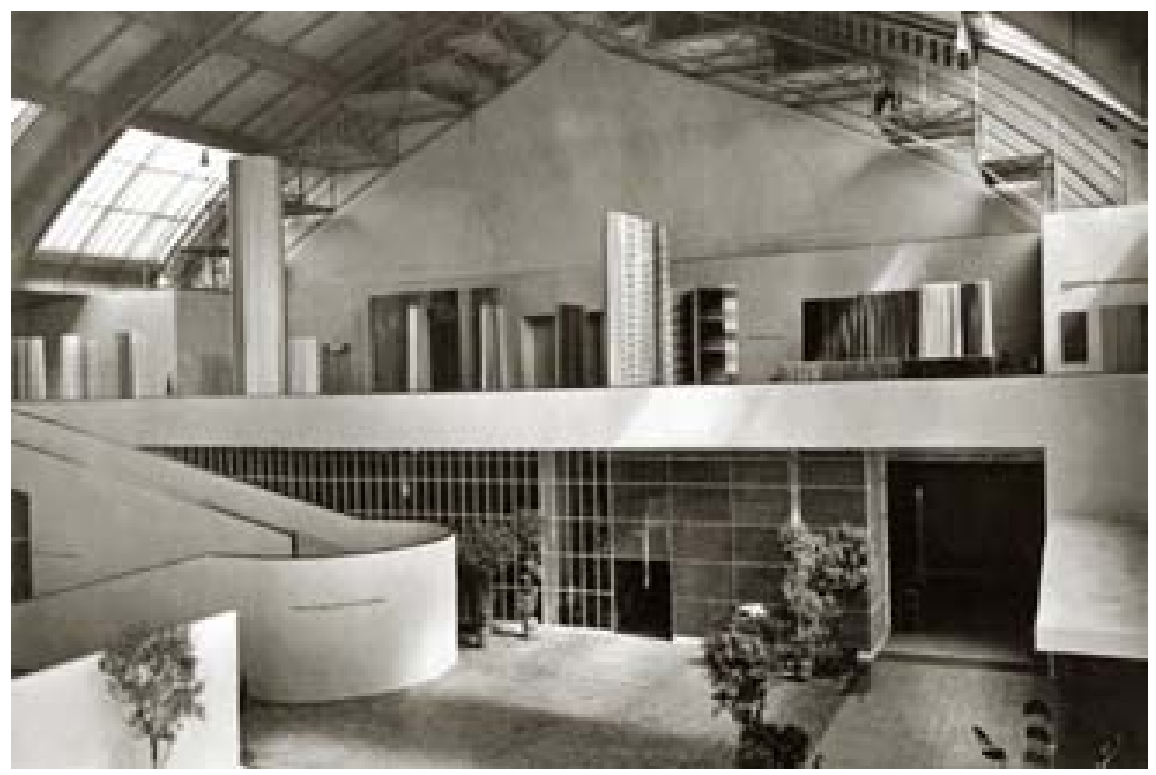

7. Muestra Die Wohnung unserer Zeit. Exposición Deutsche Bauausstellung de 1931. Diseño de Mies van der Rohe.

Fuente: McQuaid, Matilda. Lilly Reich. Designer and Architect. Museum of Modern Art, Harry N. Abrams, Inc., Nueva York, 1996.

Propiedad de The Museum of Modern Art, The Mies van der Rohe Archive, Nueva York. Fotografía realizada por Curt Rehbein.

9. "Vivienda para un matrimonio sin hijos". Muestra Die Wohnung unserer Zeit. Exposición Deutsche Bauausstellung de 1931. Diseño de Mies van der Rohe.

Fuente: The Mies van der Rohe Archive. Part I, 1910-1937. Vol. 1. Garland, Nueva York, 1986. Propiedad de The Museum of Modern Art, The Mies van der Rohe Archive, Nueva York.

8. Perspectiva de la idea inicial del conjunto de la muestra Die Wohnung unserer Zeit. Exposición Deutsche Bauausstellung de 1931. Dibujo realizado por Mies van der Rohe.

Fuente: Von Ursel, H. y T. Pavel. Barcelona Pavilion. Mies van der Rohe \& Kolbe. Architecture \& Sculpture. Jovis Verlag, Berlín, 2006. Propiedad de The Museum of Modern Art, The Mies van der Rohe Archive, Nueva York.
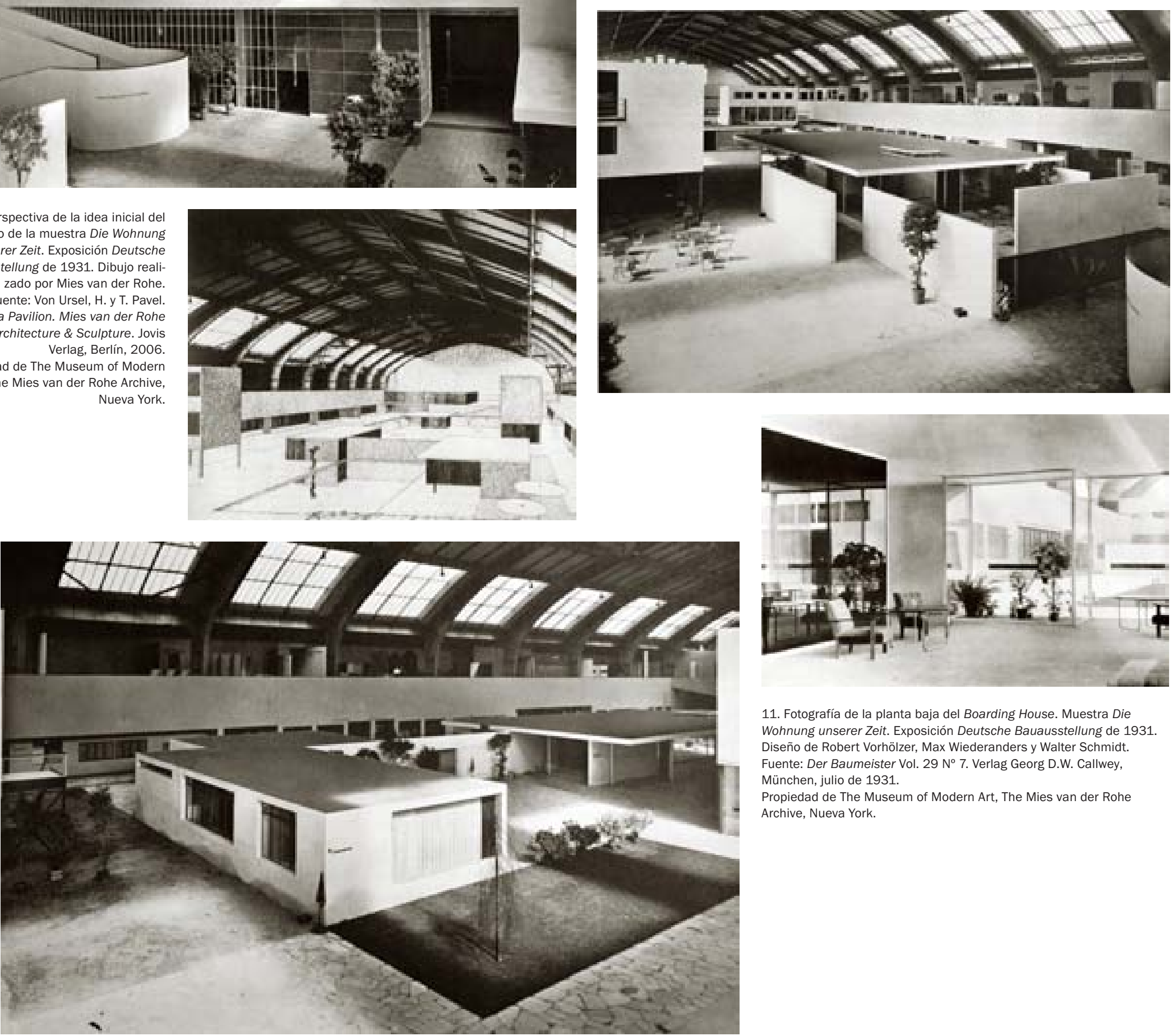

11. Fotografía de la planta baja del Boarding House. Muestra Die Wohnung unserer Zeit. Exposición Deutsche Bauausstellung de 1931. Diseño de Robert Vorhölzer, Max Wiederanders y Walter Schmidt. Fuente: Der Baumeister Vol. 29 № 7. Verlag Georg D.W. Callwey, München, julio de 1931

Propiedad de The Museum of Modern Art, The Mies van der Rohe Archive, Nueva York.

10. Vivienda en Planta Baja". Muestra Die Wohnung unserer Zeit. Exposición Deutsche Bauausstellung de 1931. Diseño de Lilly Reich.

Fuente: The Mies van der Rohe Archive. Part I, 1910-1937. Vol 1. Garland, Nueva York, 1986.

Propiedad de The Museum of Modern Art, The Mies van der Rohe Archive, Nueva York. 
Nuestro Tiempo" fuera una experiencia arquitectónica dentro de un espacio existente $y$, por tanto, presentase notables diferencias con las exposiciones residenciales con implantación territorial, la muestra fue diseñada exprofeso con fines experimentales y divulgativos, combinando la práctica arquitectónica con la teoría de una nueva manera de ver y vivir la ciudad.

Únicamente, en lo referente a la organización perimetral, Mies tuvo en consideración el diseño de Bartning. El planteamiento de la zona central fue radicalmente distinto; mientras que Bartning planteó una serie de edificios independientes, la propuesta de Mies consistía en distintas unidades residenciales, todos ellas de cubierta plana, que si bien estaban sueltas unas respecto de otras, se conectaban entre sí mediante muros y tratamientos superficiales en el plano de suelo. Adicionalmente, la barandilla maciza que rodeaba todo el perímetro de la sala actuaría como elemento unificador del conjunto. El extremo enfrentado al acceso principal de la sala era el punto por el cual se podía acceder al nivel superior, a través de una rampa que también fue un diseño añadido de Mies ${ }^{5}$ (fig. 07).

En un primer momento, Mies pensó ocupar los espacios existentes entre las viviendas con piscinas, zonas de césped y pérgolas, recreando un exterior figurado. Pero finalmente, y a pesar que el resultado final fue bastante similar a esta primera intención, los edificios se dispusieron de manera más densa, lo que provocó que el sentimiento de ligereza y amplitud del boceto se perdiera en el proyecto construido (fig. 08). Sin embargo, tanto en el croquis inicial como en la propuesta realizada, se evidencia la intención de Mies respecto a crear ciudad; una ciudad delimitada por los edificios y sus fachadas; una ciudad recorrida en dos niveles y de dos maneras, central y perimetral; una ciudad cualificada, no sólo por las volúmenes edificados, sino por los espacios urbanos y elementos arquitectónicos que interconectaban lleno y vacío, creando recorridos con acontecimientos vinculados a espacios públicos que definían, a ojos del visitante, un paisaje urbano.

La planta baja del recinto albergó un total de veintitrés viviendas, cinco de las cuales ocupaban un lugar central y protagonista; eran las viviendas de Lilly Reich, Hugo Häring, los hermanos Wassili y Hans Luckhardt, el dúplex de Otto Haesler y Karl Völkers y la vivienda unifamiliar del propio Mies van der Rohe. El resto de las casas, diseñadas por Josef Albers, Walter Gropius, Erwin Gutkind, Ludwig Hilberseimer, Marcel Breuer e incluso Wassili Kandinsky, entre otros, se dispusieron en el perímetro, bajo unas pasarelas transitables. De entre todos los proyectos destacó la "Vivienda para un matrimonio sin hijos" diseñada por Mies van der Rohe y descrita en el catálogo de la exposición como una "vivienda en planta baja con espacios de vida relacionados entre sí y habitaciones contiguas al jardín"“ (fig. o9). Efectivamente, se trataba de un edificio de una sola altura, donde el espacio era absolutamente continuo, gran deudor de la espacialidad experimentada y materializada en el Pabellón de Barcelona, su proyecto expositivo inmediatamente anterior. Al igual que en Barcelona, la vivienda estaba configurada por muros, paramentos de vidrio, soportes metálicos y una losa de cubierta, pero por el contrario, no se proyectó de manera autónoma, sino teniendo en cuenta una ordenación urbanística de conjunto; uno de los muros se extendía sin límites enlazándose con la vivienda colindante, la 'Vivienda en Planta Baja' diseñada por Lilly Reich (fig. 10). Este gesto puede considerarse un vestigio del esquema teórico miesiano, en donde todas las viviendas estarían unidas mediante planos verticales continuos, como planteara posteriormente en los proyectos urbanos de Casas Patio realizados a mediados de los años treinta.

También en el interior de la vivienda, en la escala más peque- ña, empleó los mismos mecanismos espaciales. Bajo esta filosofía, como si de un pabellón se tratase, sin emplazamiento real o cliente específico, Mies jugó con amplios espacios ininterrumpidos, sin puertas ni compartimentaciones estancas, en donde los planos verticales exentos organizaban el recorrido. Los posibles movimientos y los distintos niveles de opacidad de los paramentos hacían el espacio cambiante y desdibujaban los límites de las estancias.

Además de las viviendas unifamiliares y los expositores industriales, la muestra contó con el montaje de dos edificios plurifamiliares basados en la idea de mancomunidad. El Boarding House u Hotel de Apartamentos y el Edificio de Administración del Gobierno Prusiano. El Boarding House, como cualquier edificio de su misma tipología, contenía una serie de habitaciones sin cocina, para alojar uno o dos inquilinos. Sus arquitectos, Robert Vorhölzer, Max Wiederanders y Walter Schmidt, propusieron distintos tipos de espacios acordes con las nuevas necesidades sociales, culturales y económicas: no sólo diseñaron habitaciones dormitorio, sino también todas las zonas comunes requeridas en un edificio de esta naturaleza. Situaron en planta baja un café restaurante, un patio de encuentro y una sala de reuniones. En la planta primera y segunda organizaron las unidades habitacionales, diferenciando entre apartamentos para hombres solteros, para mujeres solteras y para parejas sin hijos (fig. 11). El edificio de dos plantas patrocinado por la Administración del Estado y el Ministerio de Finanzas Prusiano, diseñado por Otto Haesler y Karl Voelker, contenía una gran diversidad de interiores, incluyendo una pequeña sala de conciertos circular, salas de reuniones, algunos modelos de oficinas e incluso algunos ejemplos de celdas de prisión. El documento 25.107 del Archivo Mies van der Rohe, dibujado en la oficina de Mies, sugiere que el arquitecto también intervino de algún modo en el diseño de esta parcela separada.

Mies tuvo en cuenta todos los detalles de cada uno de los edificios, ya fueran viviendas unifamiliares o bloques plurifamiliares, y los orquestó en una composición conjunta donde sus fachadas actuaban en continuidad. Todas ellas formaron un conjunto homogéneo gracias al empleo de unas proporciones similares y a un número limitado de materiales: superficies blancas, lisas y con gran protagonismo del vidrio. No sólo se preocupó por utilizar un criterio común en planta baja, sino que creó también una continuidad entre las viviendas y los materiales expuestos en la galería superior, en la "Muestra de Materiales" diseñada por Lilly Reich. Las fachadas de las viviendas perimetrales se prolongaban hasta configurar el antepecho de la galería superior y la rampa de conexión entre ambos niveles. Con este sencillo gesto la fachada se convertía en rampa y a su vez, formaba parte del paisaje exterior, que terminaba por envolver el espacio de la exposición. El escenario perimetral construido a modo de "funda interior" conformaba la nueva fachada de la ciudad, dentro de la cual dispuso viviendas de todas las tipologías posibles, perfectamente entrelazadas y espacialmente bien compensadas, formando espacios abarcables y reconocibles a través del recorrido sinuoso y cuasi laberíntico del visitante. A nivel del peatón, el espectador no podía captar la magnitud de la sala, pero cuando subía a la entreplanta podía ver el conjunto arquitectónico en su totalidad. Para posibilitar esa panorámica, la percepción exterior de las unidades residenciales era abstracta y sencilla; sin embargo, mientras exteriormente las cinco

5 En el Archivo Mies van der Rohe del MoMA se conservan tres croquis de la rampa. Documentos $25.6,25.7$ y 25.8 .

6 Así lo consigna el catálogo Amtlicher Katalog und Führer. Herausgeber: Ausstellungs-, Messe- und Fremdenverkehrs-Amt der Stadt Berlin. Deutsche Bauausstellung Berlin editado en 1931. 


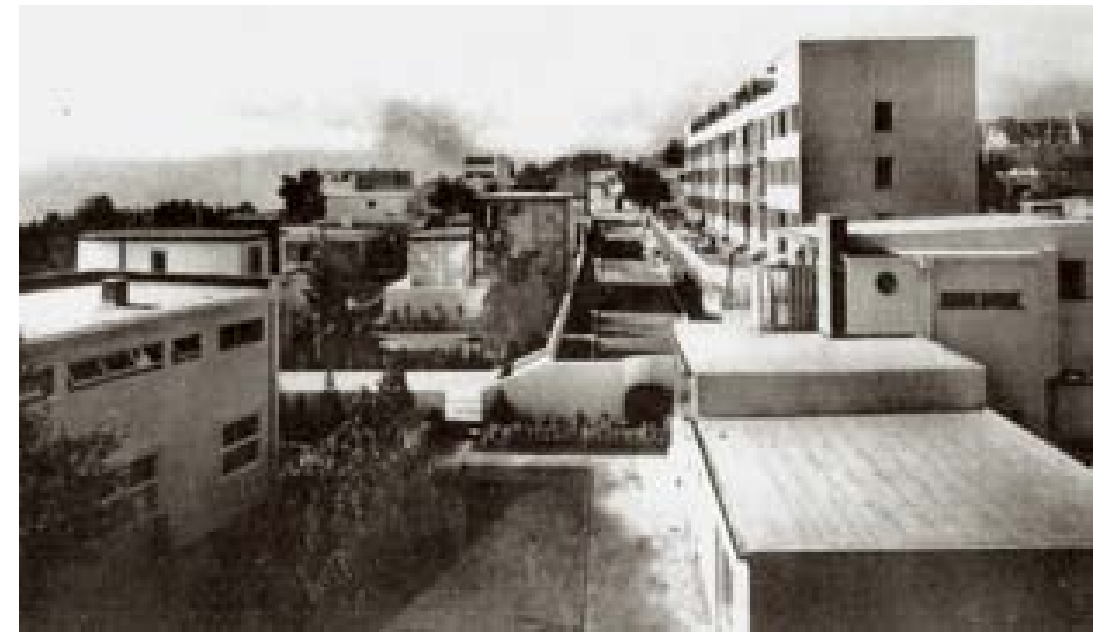

fachadas eran lisas, neutras y desprovistas no sólo de ornato sino de otros elementos constructivos -como pudiesen ser los canalones para aguas lluvia, los remates de las cubiertas o incluso carpinterías estancas- los interiores fueron detallados concienzudamente. Como en la arquitectura de las civilizaciones más antiguas, un exterior austero no eximía conseguir un interior rico, confortable y adaptable, propio de la Nueva Arquitectura.

El tratamiento del plano de suelo también ayudó a entender la continuidad espacial propia de la modernidad; pavimentos interiores y exteriores ignoraron los límites entre el espacio "de dentro" y el "de fuera". Además, la disposición de un mismo mobiliario, tanto en el interior como en el exterior, enfatizó la continuidad de la sala. Enlazó fragmentos sin relación previa, envolviéndolos en el nuevo mundo de la arquitectura que estaba por venir y absorbió cualquier elemento de la sala expositiva poniendo en valor la arquitectura futura. La continuidad espacial era parte de la modernidad, que evitaba cualquier asociación con el edificio preexistente y que confería al conjunto gran autenticidad. Fue el mismo concepto que intentó conseguir en la Weissenhoffsiedlung pero que las autoridades rechazaron por presentar posibles dificultades a la hora de definir la propiedad para vender las unidades residenciales. Mientras que en Stuttgart la condición de permanencia de las viviendas no le dio la posibilidad de enlazar unas parcelas con otras, el carácter efímero de la exposición de Berlín sí le permitió experimentar este concepto con más profundidad; consiguió desdibujar los limites existentes entre lo público y lo privado, gracias a los mecanismos del plano vertical -el muro- y el plano horizontal de pavimentos, pérgolas y recorridos. Todos estos mecanismos actuaron como elementos de unión entre las viviendas.

Las críticas hacia la "Vivienda de Nuestro Tiempo" fueron variadas $^{7}$. El gremio de arquitectos, especialmente los pertenecientes al Deutscher Werkbund, ensalzó la muestra manifestando la emoción que les había producido poder experimentar la nueva arquitectura a escala real. La crítica arquitectónica extranjera también fue muy positiva, destacando las declaraciones de Henry-Russell Hitchcock y Philip Johnson, quienes alabaron enormemente la actuación de $\mathrm{Mies}^{8}$. Sin embargo, los críticos profesionales analizaron la exposición como si se tratara de una serie de edificios reales, encontrando, en consecuencia, problemas y contradicciones. Con el público en general ocurrió algo similar. En lugar de aceptar las unidades residenciales como fragmentos de obras de arquitectura basadas en ideas experimentales, buscaron edificios tradicionales, conocidos, y no los encontraron. Las viviendas estaban inmersas en un paisaje artificial, de modo que el contexto arquitectónico no podía ser una reproducción precisa de un edificio construido en un entorno real
Mientras que en Stuttgart la condición de

permanencia de las viviendas no le dio la

posibilidad de enlazar unas parcelas con otras,

el carácter efímero de la exposición de Berlín

sí le permitió experimentar este concepto con

más profundidad; consiguió desdibujar los

limites existentes entre lo público y lo privado,

gracias a los mecanismos del plano vertical -

el muro- y el plano horizontal de pavimentos,

pérgolas y recorridos.

y con vocación de permanencia. La exposición, por el contrario, tenía que descansar en la autenticidad de la experiencia vivida y la reflexión surgida de cada uno, y no en el propio objeto en sí. Además, las viviendas fueron criticadas por presentar problemas técnicos y económicos en lugar de ser vistas desde el punto de vista ideológico y humano para el que fueron creadas.

La importancia de la muestra de Mies residía en sus implicaciones culturales, sociales y urbanísticas, presentando un nuevo estilo de vida acorde con las necesidades modernas. Además, lo hizo de un modo distinto al esperado. Fue uno de los pocos arquitectos que experimentó el urbanismo y la arquitectura desde la exposición, concibiendo la exposición fuera de los modelos convencionales del arte -museos- y la construcción -ferias comerciales-. "Exposición” o "Experimento" sugería que los proyectos no debían basarse en dar soluciones específicas a problemas prácticos, sino que se trataba de realizar una serie de arquitecturas que investigaran los aspectos que la práctica arquitectónica no permitía. Una arquitectura sin limitaciones ni restricciones. Exposiciones como "demostraciones de fuerza dirigentes que lleven a una revolución en la manera de pensar" (Van der Rohe, 1928).

\section{EPÍLOGO}

La organización de la Weissenhof estuvo muy condicionada por los planteamientos urbanísticos de unas parcelas que iban a perdurar y 


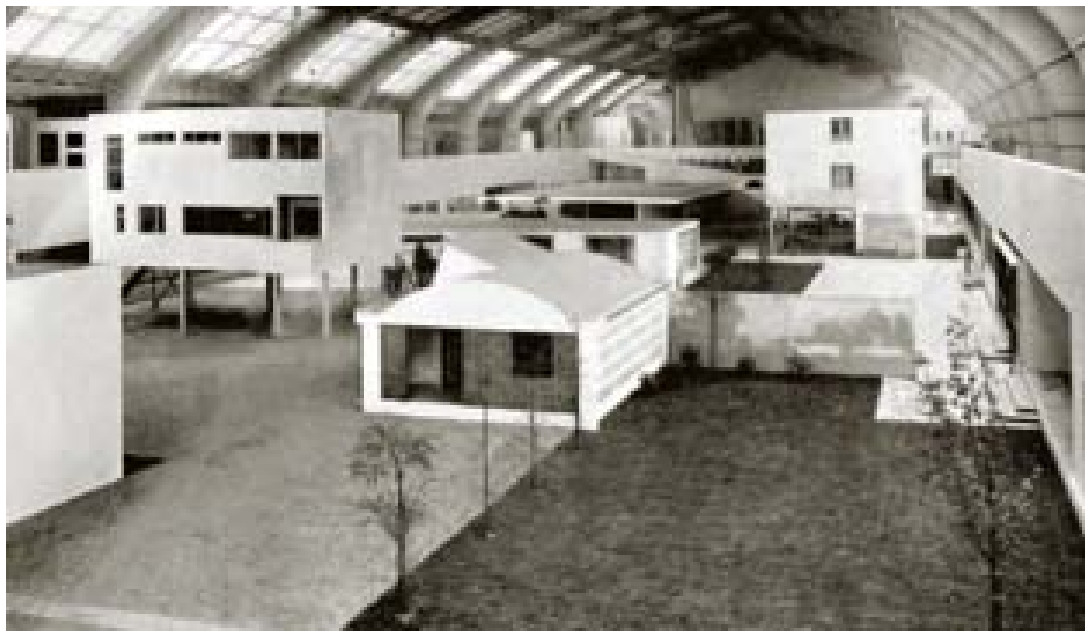

a ser habitadas. La necesidad de definir el límite entre lo privado y lo público alteró los planteamientos iniciales de Mies, mucho más conectados y abiertos. Sin embargo, en Berlín, encerrado dentro de una construcción preexistente, se liberó de aquellos condicionantes y planteó lo que entonces no pudo: viviendas que, aunque siguen siendo de distintos autores y en base a planteamientos estéticos y compositivos comunes, ahora se entrelazan con elementos construidos -pérgolas, patios, recorridos, planos que sobrepasan cubiertas y límites- que difuminan el margen entre lo público y privado, entre el interior y el exterior. Fondo y figura formaron parte de un entorno, construido por diferentes artistas, en donde se reafirmaba la identidad arquitectónica de una época por encima de la individualidad del arquitecto creador y se garantizaba la unidad y continuidad del conjunto por encima del protagonismo de la pieza (fig. 12).

Los espacios expositivos fueron para Mies el laboratorio en el que pudo ensayar las ideas y formas arquitectónicas que más tarde construiría en su arquitectura "real". Al igual que el panel curvo del Café de Terciopelo y Seda o el muro de ónice del Pabellón de Barcelona fueron los principios precedentes del espacio principal de la casa Tugendhat, la muestra Die Wohnung unsererZeit avanzó en los planteamientos relacionados con las agrupaciones residenciales investigados previamente en Stuttgart. El paisaje y los edificios se concibieron con unidad; sus límites se diluyeron, ganando importancia los espacios intersticiales que surgen de esta fusión. Los paisajes experimentales, naturales y artificiales, y los edificios expositivos, permanentes y temporales, ofrecieron a Mies la oportunidad de ensayar nuevas relaciones entre planeamiento y edificación, entre espacio público y privado, entre interior y exterior, entre mundo, comunidad e individuo. Arq

7 Hubo críticas periodísticas positivas, como la publicada en el Vossische Zeitung: "Si hoy antes del medio día vienen los invitados a Westend, la fiesta de inauguración de la gran empresa de la Exposición de Berlín, recibirán una gran impresión por el enorme trabajo all realizado y por la excelente organización de la gigantesca obra (...) Los mejores nombres de la nueva arquitectura han tomado aquí partido, Gropius, Luckhardr, Häring, la Bauhaus, Lilly Reich, Haesler en Celle, Marcel Breur, Vorhoelzer, etc". Vossische Zeitung. Berlín, 9 de mayo de 1931. También hubo críticas negativas como la escrita en el Die Weltbühne de Berlín: “Actualmente no necesitamos ninguna Exposición de la Vivienda del hombre que posee demasiado dinero". Die Weltbühne. Berlín, 4 de agosto de 1931. Wilhelm Lotz las llamó "viviendas unifamiliares exuberantes y espaciosas que no tenían nada que ver con los problemas de la vivienda convencional" (Lotz, 1937).

8 Henry-Russell Hitchcock escribió: "El descubrimiento más importante de arquitectura este verano ha sido la Exposición de la Construcción en Berlín (...) El interés principal de la exposición se encontraba en la Sala II, que había nacido completamente bajo la dirección de Mies van der Rohe. De las clases de mármol expuestas, maderas y tejidos -los cuales fueron seleccionados por Mies y dispuestos por Lilly Reich- hasta la casa de Mies en el centro de la composición, todo está dispuesto con una claridad de visión que sólo se puede alcanzar mediante un control de un único gusto positivo". Hund und Horn 1931/1932, p. 94. (Günther, 1988). Philip Johnson afirmó: “En este tipo de composición tridimensional se desafía la fotografía (...) Sólo andando a través del edificio se puede obtener una idea de su belleza" (Johnson, 1932).
Laura Lizondo | Arquitecta y Doctora Arquitecta, 2003 y 2012, Universitat Politècnica de València. Su proyecto final fue premiado por la UPV, Revista VIA y el CSCAE. En 2011 fue Visiting Scholar en Columbia University GSAPP y en 2012 fue profesora invitada en University of Stavanger. Actualmente es profesora del Departamento de Proyectos Arquitectónicos en la Escola Tècnica Superior d'Arquitectura de la Universitat Politècnica de València e investigadora del grupo "Intervención en la Arquitectura Monumental e Histórica".

José Santatecla-Fayos | Arquitecto y Doctor Arquitecto, 1986 y 2005, Universitat Politècnica de València. Su obra construida ha sido premiada en diferentes concursos en España y ha sido profesor invitado en varias universidades en cursos sobre Teoría y Pensamiento. Actualmente es profesor del Departamento de Proyectos Arquitectónicos de la Escola Tècnica Superior d'Arquitectura de la Universitat Politècnica de València e investigador del grupo "Arte y arquitectura contemporánea".

Ignacio Bosch-Reig | Arquitecto y Doctor Arquitecto, 1972 y 1995, Universitat Politècnica de València. En 1995 recibió el Premio Comisión Europea "Intervention Pilot Project Heritage" y en 2006 el premio Europa Nostra en Conservación del Patrimonio. Entre 2000 y 2005 fue directo del Instituto de Restauración del Patrimonio de la UPV, donde también fue director de la Escola Tècnica Superior d'Arquitectura entre 2003 y 2008. Desde 2002 es director de la revista R\&R. Actualmente es Catedrático del Departamento de Proyectos Arquitectónicos de la Escola Tècnica Superior d'Arquitectura de la Universitat Politècnica de València.

\section{Bibliografía}

AA.VV. Amtlicher Katalog und Führer. Herausgeber: Ausstellungs-, Messe- und FremdenverkehrsAmt der Stadt Berlin. Deutsche Bauausstellung Berlin 1931. Bauwelt-Verlag, Berlín, 1931. BAUDELAIRE, Charles. "Le Peintre de la vie moderne". Le Figaro del 26, 29 de noviembre y del 3 de diciembre, París, 1863

BENJAMIN, Walter. Das Kunstwerk im Zeitalter seiner technischen Reproduzierbarkeit. Félix Alcan, París, 1936.

COLOMINA, Beatriz. "La casa de Mies: exhibicionismo y coleccionismo". 2G № 48-49 Mies van der Rohe. Casas. Editorial Gustavo Gili, Barcelona, 2009.

DREXLER, Arthur y Franz SCHULZE. Mies van der Rohe Archive 1910-1937. Museum of Modern Art, Nueva York, 1986.

GÜNTHER, Sonja. Lilly Reich 1885-1947. Innenarchitektin. Designerin. Ausstellungsgestalterin. Deutsche Verlags-Anstalt, Stuttgart, 1988.

HONEY, Sandra. Mies van der Rohe: European Works. Architectural Monographs. Academy Editions, Londres; : St. Martin's Press, Nueva York, 1986, p. 11-25.

JOHNSON, Philip. "The Berlin Building Exposition of 1931". T-Square 2 № 1. Enero de 1932, p. 18. KIRSCH, Karin. The Weissenhofsiedlung. Experimental Housing Built for the Deutscher Werkbund, Stuttgart, 1927. Rizzoli, Nueva York, 1986.

LOTZ, Wilhelm: “Die Halle II auf der Bauausstellung”. Die Form Vol. 6 № 7. Verlag Hermann Beckendorf, Berlín, 1931.

MCQUAID, Matilda: Lilly Reich. Designer and Architect. Museum of Modern Art, Harry N. Abrams Inc., Nueva York, 1996.

MILLER, Wallis. Tangible Ideas: Architecture and the Public at the 1931 German Building Exhibition in Berlin. Tesis Doctoral, Princeton University, Nueva York, 1999.

MILLER, Wallis. "Mies and Exhibitions". Mies in Berlin. Museum of Modern Art, Harry N. Abrams Inc., Nueva York, 2001, p. 338-350.

MILLER, Wallis. "Cultures of Display. Exhibiting Architecture in Berlin, 1880-1931". Architecture and Authorship. Black Dog Press, Londres, 2007, p. 97-107.

NEUMEYER, Fritz. La palabra sin artificio. Reflexiones sobre arquitectura 1922/1968. El croquis Editorial, Madrid, 1986.

PÉREZ OYARZUN, Fernando. “Pabellón Philips. Bruselas, Bélgica”. Revista ARQ №63. Ediciones ARQ, Santiago, 2006, p. 54-59.

POMMER, Richard y Christian F. OTTO. Weissenhof 1927 and the Modern Movement in Architecture. University Press, Chicago, 1991.

SCHULZE, Franz. Mies Van der Rohe. A critical biography. University of Chicago Press, Chicago y Londres, 1985.

STANKARD, Mark. "Re-covering Mies van der Rohe's Weissenhof: The Ultimate Surface”. Journal of Architectural Education, May 2002, no. 54-55, p. 247-256.

VAN DER ROHE, Mies. "Zum Thema: Ausstellungen“. Die Form Vol. 3 № 4 . Verlag Hermann Beckendorf, Berlin, 1928, p. 121. 